\title{
A house with many rooms: how the heart got its chambers with foxn4
}

\author{
Ethan David Cohen ${ }^{1}$ and Edward E. Morrisey ${ }^{1,2,3,4}$ \\ ${ }^{1}$ Cardiovascular Institute, University of Pennsylvania, Philadelphia, Pennsylvania 19104, USA; ${ }^{2}$ Institute for Regenerative \\ Medicine, University of Pennsylvania, Philadelphia, Pennsylvania 19104, USA; ${ }^{3}$ Department of Medicine and Department of \\ Cell and Developmental Biology, University of Pennsylvania, Philadelphia, Pennsylvania 19104, USA
}

The vertebrate heart is initially formed from two lateral domains of cardiogenic mesoderm that fuse at the ventral midline to form a simple heart tube that is similar to the hearts or dorsal vessels of invertebrates. As development proceeds, this initial heart tube undergoes a series of morphogenetic rearrangements that subdivides the heart into the distinct chambers found in adult vertebrates. In this issue of Genes \& Development, Chi et al. (2008) describe a novel transcriptional pathway required for separation of the outflow (ventricular) portion from the inflow (atrial) portion of the two-chambered zebrafish heart. This separation takes place at the atrioventricular (AV) canal, which forms the valves separating the ventricular and atrial compartments required for unidirectional blood flow, as well as the electrical conduction system required for proper cardiac rhythm. Chi et al. (2008) identify a member of the forkhead box (fox) family of transcription factors, foxn4, which works in concert with $t b x 5$ to direct the development of the AV boundary by regulating the expression of $t b \times 2 b$. This study sheds new light on how the AV canal myocardium became specialized during vertebrate evolution to perform its function as both the gatekeeper of blood flow and regulator of cardiac rhythm.

\section{Development and function of the AV canal and foxn4}

The basic design of the cardiovascular system changes dramatically during evolution to accommodate the increased blood flow and pressure found in vertebrates. In Drosophila, the peristaltic contraction of a simple myocardial tube promotes the circulation of hemolymph, the insect version of blood, within an open circulatory system. While this is sufficient for invertebrate species, which have small surface area-to-volume ratios and a relatively low demand for oxygenated blood, vertebrate species require more complex circulatory loops that provide directional flow of oxygenated blood from gas exchange organs such as lungs or gills to the rest of the

${ }^{4}$ Corresponding author.

E-MAIL emorrise@mail.med.upenn.edu; FAX (215) 573-2094.

Article is online at http://www.genesdev.org/cgi/doi/10.1101/gad.1662408. organism, followed by the subsequent return of deoxygenated blood to the heart. The simplest form of this unidirectional blood flow is seen in the two-chambered fish heart. Blood returning from the body first enters a receiving chamber or atria, which then contracts to force blood into a larger pumping chamber or ventricle through a valve structure that is specialized to prevent retrograde flow. After filling, the ventricle contracts, forcing blood out of the heart into the aorta where it is first delivered to the gills for oxygenation before being circulated to the rest of the body.

The AV boundary, which separates the atrial and ventricular compartments, plays at least three critical roles in the establishment of unidirectional flow through the heart. First, both myocardial and endocardial cells within the AV boundary undergo characteristic cell shape changes that cause the heart tube to bend in this region and form a physical constriction between the atrial and ventricular compartments. Second, the endocardial cells within the AV boundary delaminate and migrate into the lumen, where they form the endocardial cushions that later differentiate into the AV valve. Third, while the waves of electrical stimulation and subsequent contractility begin at the inflow poles of both vertebrate and invertebrate hearts, cells within the AV canal of the vertebrate heart have fewer gap junctions and slow the transfer of electrical excitation, thus allowing for the sequential contraction of the atrial and ventricular compartments. Finally, it should be noted that AV canal defects, including valvular defects, comprise a large and important group of human congenital heart diseases whose underlying molecular etiology is still poorly understood.

In a study appearing in this issue of Genes \& Development, Chi et al. (2008) report that the zebrafish mutant, slipjig (sli), exhibit severe defects in AV canal development, leading to cardiovascular failure in these mutants. Chi et al. (2008) mapped the mutation to the zebrafish ortholog of the foxn 4 gene, a member of the large fox family of transcription factors, which play key roles in regulation of cell specification, differentiation, and proliferation during development. Although the linear heart tube develops normally in sli mutants, neither myocardial nor endocardial cells undergo the characteristic cell 
shape changes within the AV boundary and the heart tube fails to loop. Furthermore, the endocardial cushions as well as their derivative valve structures develop abnormally and the normal delay in electrical conduction at the AV boundary fails to develop so that the heart continues to display peristaltic beating similar to that of the early heart tube in sli mutants. Taken together, these data suggest that sli/foxn4 is required for AV boundary specification in the zebrafish heart.

Consistent with foxn 4 being required for zebrafish AV canal specification, several genes, including $t b \times 2 b$, expressed in the AV boundary of wild-type hearts, fail to localize in sli mutants (Chi et al. 2008). Tbx2 was previously shown to be required for AV canal and outflow tract development in the mouse (Harrelson et al. 2004). In the current report by Chi et al. (2008), morpholino knockdown of $t b \times 2 b$ in zebrafish phenocopies the loss of AV boundary formation and the defects with electrical conduction observed in sli mutants. sli mutants display expanded expression of AV canal markers such as bmp4 and versican and expanded expression of the endocardial marker notch $1 b$, all pointing to a loss of AV canal myocardial and endocardial specification or differentiation (Chi et al. 2008). Furthermore, the $t b \times 2 b$ promoter contains conserved binding sites for foxn 4 and another T-box family transcription factor tbx5. foxn 4 and $t b x 5$ both bind directly to the $t b \times 2 b$ promoter in electrophoretic mobility shift assays, and mutating the binding sites for either foxn 4 or $t b x 5$ abolishes the AV canal specific expression of GFP reporters under the control of the $t b \times 2 b$ promoter in transgenic zebrafish embryos. Chi et al. (2008) went even further to draw the link between foxn 4 and $t b \times 2 b$ by performing morpholino knockdowns of $t b \times 2 b$. These morphants exhibited similar defects in AV canal formation and conduction delay as sli/foxn4 mutants. The authors were rigorous in their studies and attempted to rescue sli/foxn4 mutants with overexpression of $t b \times 2 b$. Although this did rescue the sli/foxn $4 \mathrm{AV}$ canal phenotype in a majority of embryos, such overexpression resulted in AV canal defects even in wild-type embryos, limiting the usefulness of this approach. Taken together, these data indicate that foxn 4 and $t b \times 5$ act in concert to directly regulate $t b \times 2 b$ expression at the $\mathrm{AV}$ boundary.

Although other mutations have been shown to disrupt AV canal development, the zebrafish sli/foxn4 mutation is unique in that it specifically ablates AV canal specification without disrupting myocardial development, and the initial linear heart tube appears to be unaffected. The report by Chi et al. (2008) therefore raises the intriguing possibility that foxn 4 was harnessed to direct the expression of targets, such as $t b \times 2 b$, to promote the specialized differentiation of the AV canal myocardium during the evolutionary jump from the simple heart tube found in invertebrates to the multichambered hearts of vertebrates. While the Drosophila heart consists of a linear tube, there is differential gene expression along its anterior-posterior axis, and two Drosophila tbx20 homologs recently have been shown to be required for this polarized gene expression (Miskolczi-McCallum et al. 2005;
Qian et al. 2005; Reim et al. 2005). A Fox gene has not yet been implicated in early Drosophila cardiac development, further supporting the hypothesis that members of this gene family including foxn 4 have been recruited to help define the AV boundary in vertebrates. The expression of foxn4 to the AV boundary and its regulation of $t b \times 2 b$ therefore may have allowed a partially pre-existing set of positional cues, in the case of $t b x$ factors, to be modified in order to define the AV boundary for its specialized functions (Fig. 1).

\section{Fox genes and cardiac development}

The current findings by Chi et al. (2008) are not the first to implicate Fox genes in the regulation of cardiac development. For example, Foxp1 mutants display defects in the endocardial-mesenchymal transition (EMT) process that drives AV cushion development, in addition to defects in myocardial proliferation and differentiation and outflow tract septation (Wang et al. 2004). Foxc1/c2 also regulate cardiac outflow tract septation, in part, through regulation of $T b x 1$, providing an
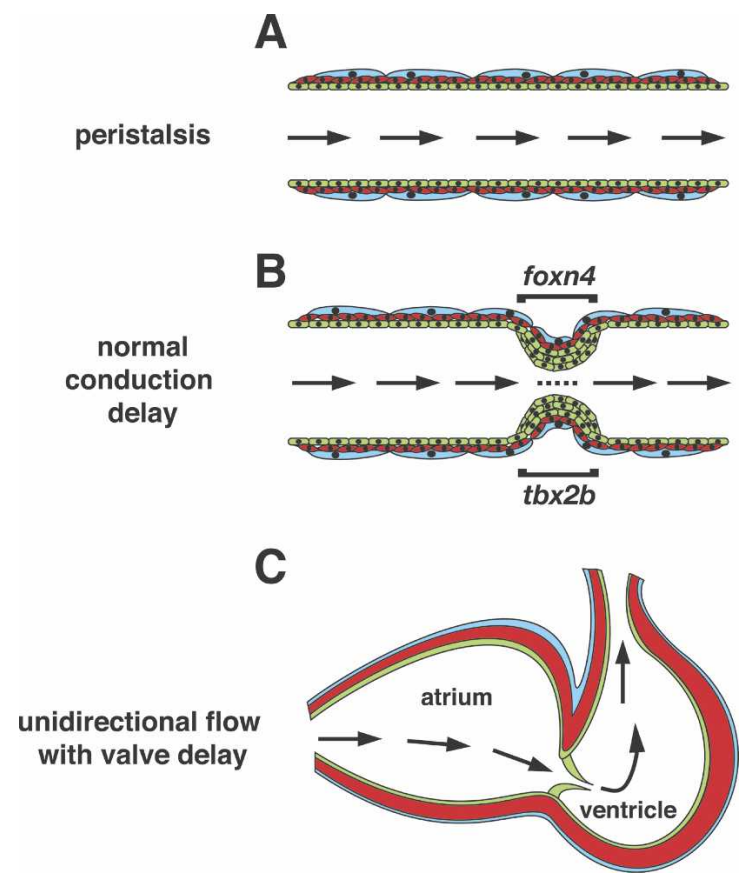

Figure 1. Development of a simple vessel into a two-chambered heart using foxn4-tbx2b. (A) The zebrafish heart begins as a simple vessel containing myocardial (red), epicardial (blue), and endocardial (green) cells. The zebrafish heart resembles the dorsal vessel in insects at this stage, except for the presence of epicardial cells in the vertebrate heart, and pumps blood primarily by peristaltic action. (B) Upon expression of foxn4, which activates $t b \times 2 b$ expression, the endocardial cells ingress inward, forming the AV canal, forming a valve, and causing a conduction delay required for setting up a unidirectional circulation. (C) Finally, the mature two-chambered heart with mature valves can circulate blood with high efficiency in a closed system. Arrows indicate both blood flow and direction of electrical conduction in the developing zebrafish heart. 
important example of a Fox-Tbx link in mammalian cardiac development (Yamagishi et al. 2003; Seo and Kume 2006). Foxp1, Foxc1, and Foxc2 are all expressed in the cardiac neural crest as well as in the secondary heart field (SHF), suggesting that these factors regulate important aspects of these cell lineages that are known to be required for proper outflow tract and right heart development (Shu et al. 2001; Yamagishi et al. 2003; Wang et al. 2004; Seo and Kume 2006). Thus, this gene family serves several critical roles in regulating diverse aspects of cardiac development, providing precedence for a role for foxn4 in cardiac development.

A Foxn4-null mutation has been reported in the mouse (Christoffels et al. 2004). Although the investigators were primarily interested in the role for Foxn4 in retinal neurogenesis, they did observe that Foxn 4 mutants died perinataly (Christoffels et al. 2004). Thus, it remains to be determined if Foxn4 is required for AV boundary specification in the mouse. The current report by Chi et al. (2008) should spur an interest in re-examination of these mice for AV canal defects. Given the presence of multiple Fox genes in the heart, and in particular in the $\mathrm{AV}$ canal region, any future work must take into account the increased complexity; i.e., redundancy among these factors that likely exists in higher vertebrates required for directing morphogenesis of a more complex AV canal. This factor, among others, is one of the strengths of using the zebrafish system, as often there is less redundancy in the gene networks that regulate processes such as AV canal development.

Fox proteins bind to a conserved DNA-binding site through a highly conserved winged-helix DNA-binding domain (for review, see Lehmann et al. 2003). Fox genes have been further divided into subfamilies based on sequence homology outside of the DNA-binding domain (Kaestner et al. 2000). Members within these subfamilies often act redundantly in cell types where they are coexpressed. However, different subfamilies have different transcriptional regulatory characteristics. For example, Foxc1/c2 are thought to be activators of gene transcription while Foxp1 has been shown to repress gene transcription (Shu et al. 2001; Yamagishi et al. 2003). These data lead to an interesting hypothesis that there is a balance of Fox activation and repression that helps pattern the developing AV canal. Such a hypothesis is testable with additional work and the zebrafish model system, which is highly amenable to multiple gene knockdowns via morpholino oligonucleotide inhibition, will undoubtedly prove valuable for such studies.

\section{A Fox-Tbx pathway in cardiac development}

In their analysis of the sli/foxn 4 phenotype, Chi et al. (2008) showed that tbx $2 \mathrm{~b}$ expression was lost in the AV canal of the sli mutants. Tbx genes have been known for many years to play key roles in cardiac development including AV canal, ventricular growth, and outflow tract septation. In mice, $T b \times 2$ is required for proper AV canal development in part by repressing atrial and ventricular chamber-specific gene expression programs, keeping the
AV canal myocardium in a more plastic state (Habets et al. 2002; Christoffels et al. 2004; Harrelson et al. 2004; Cai et al. 2005). Moreover, Tbx2 negatively regulates myocardial proliferation through repression of $\mathrm{N}$-myc, which is required for myocardial proliferation (Cai et al. 2005). Thus, as with many other critical differentiation factors, Tbx2 appears to promote AV myocardial differentiation at the expense of proliferation. Loss of $t b \times 2 b$ expression in sli/foxn4 mutants may lead to increased proliferation in the AV canal, which could disrupt $\mathrm{AV}$ morphogenesis through improper expansion of AV myocardium. This might also explain the expansion of AV markers into the atria and ventricles including notch1b, bmp4, and versican.

Fox genes have been shown to regulate other Tbx genes during cardiac development. Yamagishi et al. (2003) showed that Tbxl expression in the pharyngeal endoderm and head mesenchyme is regulated by a Foxdependent enhancer that is also sonic hedgehog (Shh)responsive. In these studies, Shh was also required for expression of Foxc1/c2 in the head mesenchyme, implicating a transcriptional loop where Tbxl is regulated by Foxc1/c2, which in turn are regulated by Shh, leading to proper development of tissues deriving from the head mesenchyme including the SHF. Shh mutants have defects related to improper remodeling of branchial arch arteries, but there are no reports of specific defects in $\mathrm{AV}$ canal development outside of the presence of ventricular septal defects and malrotation of the outflow tract, all of which could be attributed to the obvious defects in the cardiac neural crest (Washington Smoak et al. 2005). Although Chi et al. (2008) did not look at Shh expression or activity in the sli/foxn4 mutants, it would be important to do so, since disruption of the Fox-Tbx axis of gene transcription may lead to loss of Shh in critical regions of the developing heart leading to the observed phenotype. However, since the role for neural crest and the actual existence of the SHF in zebrafish is debatable, the role for a fox-tbx axis may be restricted to the AV canal or primary myocardial cell lineages in zebrafish.

Using sophisticated imaging techniques, Chi et al. (2008) showed that sli/foxn4 mutants had an absence of the critical conduction delay required for synchronous unidirectional blood flow. Although much of this could be attributed to the morphological defects, there is precedence for the role of Tbx genes in regulation of the cardiac conduction system. Tbx2 and Tbx3 have been shown to repress expression of connexins 40 and 43, which are gap junction proteins important for proper conduction system function (Christoffels et al. 2004). In contrast, Tbx 5 activates expression of connexins 40 and 43 (Bruneau et al. 2001; Hiroi et al. 2001). Moreover, patients with Holt-Oram syndrome, which is caused by mutations in TBX5, have severe conduction system anomalies as do Tbx5 haploinsufficient mice (Basson et al. 1997; Bruneau et al. 2001). Thus, it will be important to determine whether haploinsufficient sli/foxn4 zebrafish or Foxn 4 mouse mutants exhibit conduction system defects. This would help to directly link the foxn4$t b \times 2 b$ axis in regulation of conduction system develop- 
ment, and provide an important precedent for future studies exploring the role of other Fox gene mutants in conduction system development.

\section{Fine-tuning AV canal development: implications for higher vertebrates}

The present report from Chi et al. (2008) underscores the relationship between Fox and Tbx transcription factors in cardiac development. If the increasing complexity of the heart was driven in part by the acquisition of additional transcriptional and signaling networks required to properly pattern the additional chambers and regions, then the finding that a new Fox gene is involved in one of the most important early steps in cardiac chamber evolution is indeed important. However, several questions remain, including: How did additional chambers get added during evolution? Part of the answer could be the evolution of the SHF, which generates portions of the right side of the heart including parts of the AV canal and outflow tract (for review, see Kelly and Buckingham 2002). Acquiring the SHF may have lead to the formation of additional chambers (right ventricle and right atria) and septation of the outflow tract, thus allowing the heart to further direct blood flow to the lungs in mammals that have a much higher metabolism than lower vertebrates, requiring more efficient circulatory perfusion. It is also important to consider the fact that in humans there are syndromes where either the right or left side of the heart is preferentially affected, including valve development. In Ebstein's anomaly, the tricuspid valve, which is the AV valve for the right ventricle, is malformed while the mitral valve, located in the AV region of the left ventricle, is relatively unaffected (for review, see Gurvitz and Stout 2007; Paranon and Acar 2008). Such left-right-specific defects may be indicative of additional molecular programs regulating left versus right AV canal development in mammals.

Future studies will be important in defining additional factors required for AV canal morphogenesis. Signaling pathways such as Wnt and BMP have been implicated in regulation of AV canal development, and whether FoxTbx factors act upstream of or downstream from these pathways is sure to be a point of future investigations. Moreover, the fact that there are likely more Fox factors expressed in and important for mammalian AV canal development will likely require complex mouse genetics to define the full repertoire of Fox gene function in AV canal morphogenesis. Although these studies will require much time and effort, they will likely reveal the elegant complexity and importance of this process. More importantly, they will help to define the molecular mechanism of an important cause of congenital heart disease in humans.

Although the heart started as a simple tube contracting in a peristaltic manner, evolution has generated a remarkable organ with many chambers, each required for the high demands of mammalian metabolism. With the identification of a foxn4-tbx2b pathway for developmental regulation of that gate keeper of unidirectional flow, the AV canal, we now have a better understanding of how the heart added new "rooms" to increase its efficiency as a pump in a closed circulatory system.

\section{Acknowledgments}

We thank members of the Morrisey laboratory for insightful comments. We are supported by grants from the National Institutes of Health and American Heart Association.

\section{References}

Basson, C.T., Bachinsky, D.R., Lin, R.C., Levi, T., Elkins, J.A., Soults, J., Grayzel, D., Kroumpouzou, E., Traill, T.A., Leblanc-Straceski, J., et al. 1997. Mutations in human TBX5 [corrected] cause limb and cardiac malformation in HoltOram syndrome. Nat. Genet. 15: 30-35.

Bruneau, B.G., Nemer, G., Schmitt, J.P., Charron, F., Robitaille, L., Caron, S., Conner, D.A., Gessler, M., Nemer, M., Seidman, C.E., et al. 2001. A murine model of Holt-Oram syndrome defines roles of the T-box transcription factor Tbx5 in cardiogenesis and disease. Cell 106: 709-721.

Cai, C.L., Zhou, W., Yang, L., Bu, L., Qyang, Y., Zhang, X., Li, X., Rosenfeld, M.G., Chen, J., and Evans, S. 2005. T-box genes coordinate regional rates of proliferation and regional specification during cardiogenesis. Development 132: 24752487.

Chi, N.C., Shaw, R.M., De Val, S., Kang, G., Jan, L.Y., Black, B.L., and Stainier, D.Y.R. 2008. Foxn4 directly regulates $t b \times 2 b$ expression and atrioventricular canal formation. Genes \& Dev. (this issue), doi: 10.1101/gad.1629408.

Christoffels, V.M., Hoogaars, W.M., Tessari, A., Clout, D.E., Moorman, A.F., and Campione, M. 2004. T-box transcription factor Tbx2 represses differentiation and formation of the cardiac chambers. Dev. Dyn. 229: 763-770.

Gurvitz, M. and Stout, K. 2007. Ebstein's anomaly of the tricuspid valve. Curr. Cardiol. Rep. 9: 336-342.

Habets, P.E., Moorman, A.F., Clout, D.E., van Roon, M.A., Lingbeek, M., van Lohuizen, M., Campione, M., and Christoffels, V.M. 2002. Cooperative action of Tbx2 and Nkx2.5 inhibits ANF expression in the atrioventricular canal: Implications for cardiac chamber formation. Genes \& Dev. 16: 1234-1246.

Harrelson, Z., Kelly, R.G., Goldin, S.N., Gibson-Brown, J.J., Bollag, R.J., Silver, L.M., and Papaioannou, V.E. 2004. Tbx2 is essential for patterning the atrioventricular canal and for morphogenesis of the outflow tract during heart development. Development 131: 5041-5052.

Hiroi, Y., Kudoh, S., Monzen, K., Ikeda, Y., Yazaki, Y., Nagai, R., and Komuro, I. 2001. Tbx5 associates with Nkx2-5 and synergistically promotes cardiomyocyte differentiation. Nat. Genet. 28: 276-280.

Kaestner, K.H., Knochel, W., and Martinez, D.E. 2000. Unified nomenclature for the winged helix/forkhead transcription factors. Genes \& Dev. 14: 142-146.

Kelly, R.G. and Buckingham, M.E. 2002. The anterior heartforming field: Voyage to the arterial pole of the heart. Trends Genet. 18: 210-216.

Lehmann, O.J., Sowden, J.C., Carlsson, P., Jordan, T., and Bhattacharya, S.S. 2003. Fox's in development and disease. Trends Genet. 19: 339-344.

Miskolczi-McCallum, C.M., Scavetta, R.J., Svendsen, P.C., Soanes, K.H., and Brook, W.J. 2005. The Drosophila melanogaster T-box genes midline and $\mathrm{H} 15$ are conserved regu- 
Cohen and Morrisey

lators of heart development. Dev. Biol. 278: 459-472.

Paranon, S. and Acar, P. 2008. Ebstein's anomaly of the tricuspid valve: From fetus to adult: Congenital heart disease. Heart 94: 237-243.

Qian, L., Liu, J., and Bodmer, R. 2005. Neuromancer Tbx20related genes (H15/midline) promote cell fate specification and morphogenesis of the Drosophila heart. Dev. Biol. 279: 509-524.

Reim, I., Mohler, J.P., and Frasch, M. 2005. Tbx20-related genes, mid and $\mathrm{H} 15$, are required for tinman expression, proper patterning, and normal differentiation of cardioblasts in Drosophila. Mech. Dev. 122: 1056-1069.

Seo, S. and Kume, T. 2006. Forkhead transcription factors, Foxc1 and Foxc2, are required for the morphogenesis of the cardiac outflow tract. Dev. Biol. 296: 421-436.

Shu, W., Yang, H., Zhang, L., Lu, M.M., and Morrisey, E.E. 2001. Characterization of a new subfamily of winged-helix/forkhead (Fox) genes that are expressed in the lung and act as transcriptional repressors. J. Biol. Chem. 276: 27488-27497.

Wang, B., Weidenfeld, J., Lu, M.M., Maika, S., Kuziel, W.A., Morrisey, E.E., and Tucker, P.W. 2004. Foxp1 regulates cardiac outflow tract, endocardial cushion morphogenesis and myocyte proliferation and maturation. Development 131: $4477-4487$.

Washington Smoak, I., Byrd, N.A., Abu-Issa, R., Goddeeris, M.M., Anderson, R., Morris, J., Yamamura, K., Klingensmith, J., and Meyers, E.N. 2005. Sonic hedgehog is required for cardiac outflow tract and neural crest cell development. Dev. Biol. 283: 357-372.

Yamagishi, H., Maeda, J., Hu, T., McAnally, J., Conway, S.J., Kume, T., Meyers, E.N., Yamagishi, C., and Srivastava, D. 2003. Tbx1 is regulated by tissue-specific forkhead proteins through a common Sonic hedgehog-responsive enhancer. Genes \& Dev. 17: 269-281. 


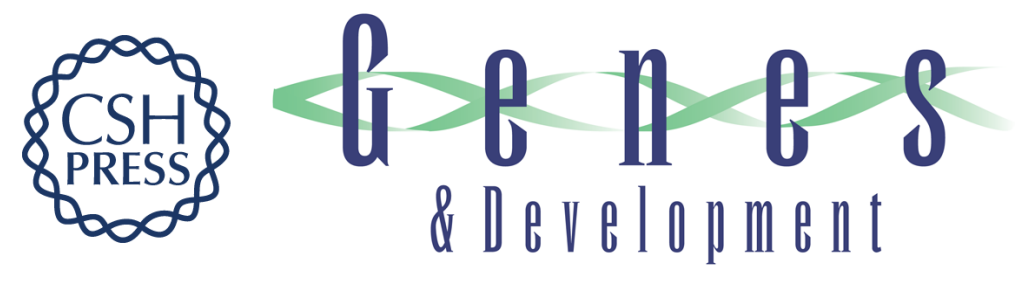

\section{A house with many rooms: how the heart got its chambers with foxn 4}

Ethan David Cohen and Edward E. Morrisey

Genes Dev. 2008, 22:

Access the most recent version at doi:10.1101/gad.1662408

Related Content Foxn4 directly regulates tbx2b expression and atrioventricular canal formation Neil C. Chi, Robin M. Shaw, Sarah De Val, et al.

Genes Dev. March , 2008 22: 734-739

References This article cites 21 articles, 9 of which can be accessed free at: http://genesdev.cshlp.org/content/22/6/706.full.html\#ref-list-1

Articles cited in:

http://genesdev.cshlp.org/content/22/6/706.full.htm|\#related-urls

\section{License}

Email Alerting

Receive free email alerts when new articles cite this article - sign up in the box at the top Service 